\title{
Letter to the Editor concerning "Relationship between preoperative serum rapid turnover proteins and early-stage surgical wound infection after spine surgery" by D. Kudo et al. (Eur Spine J; 2016: DOI 10.1007/s00586-016-4855-z)
}

\author{
Jie Weng ${ }^{1}\left[\mathrm{He} \mathrm{Wu}^{1} \cdot\right.$ Zhiyi Wang $^{1,2}$
}

Received: 14 October 2017 / Accepted: 22 March 2018 / Published online: 29 March 2018

(c) Springer-Verlag GmbH Germany, part of Springer Nature 2018

\section{Dear Editor,}

We read the article conducted by Kudo et al., which was published in European Spine Journal in 2016 [1]. The authors tried to investigate the relationship between preoperative serum rapid turnover proteins (RTPs) and postoperative surgical site infection (SSI). This study was valuable. However, some methodologic issues should be noted.

First, all data are presented as means with standard deviation (SD) in this study. We know that mean \pm SD is used in normality distribution or a Gaussian distribution $[2,3]$. The normality of continuous variables was ignored by the authors (some continuous variables were not normality distribution obviously in Table 1.

We suggest that the author conduct a normality assumption test first. Medians and interquartile ranges are more appropriate for abnormal distribution.

The potential factors related to early-stage surgical wound infection after spine surgery were identified by univariate and multivariate logistic regression analysis. First, bivariate correlations among variables should be assessed to identify potential multicollinearity before multivariate logistic regression analysis; Second, multivariate logistic regression model is determined using backward stepwise selection for those variables found to be associated with factors on univariate analysis with $p$-values less than 0.2 . In the study conducted by Kudo and colleagues, bivariate correlation

Zhiyi Wang

wzy1063@126.com

1 Department of Emergency Medicine, The Second Affiliated Hospital and Yuying Children's Hospital of Wenzhou Medical University, Wenzhou 325000, China

2 Department of General Practice, The Second Affiliated Hospital and Yuying Children's Hospital of Wenzhou Medical University, Wenzhou 325000, China between explanatory variables has not been assessed, and all of the variables from the univariate analysis were imported into the multivariate logistic regression analysis, this may lead to overparameterization [4]. The authors indicated that a low prealbumin level is a possible risk factor for earlystage SSI in spine surgery, and operative time was the most important risk factor for SSI. However, the prediction models need to be validated through using some appropriate methods such as cross-validation and bootstrapping, otherwise, these results would be optimistic interpretation [5].

\section{Compliance with ethical standards}

Conflict of interest None of the authors has any potential conflict of interest.

\section{References}

1. Kudo D, Miyakoshi N, Hongo M, Kasukawa Y, Ishikawa Y, Mizutani T, Shimada Y (2016) Relationship between preoperative serum rapid turnover proteins and early-stage surgical wound infection after spine surgery. Eur Spine J: https://doi.org/10.1007/ s00586-016-4855-Z

2. Öztuna D, Elhan AH, Tüccar E (2006) Investigation of four different normality tests in terms of type 1 error rate and power under different distributions. Turk J Med Sci 36:171-176

3. Weng J, Wu H, Xu Z, Xi H, Chen C, Chen D, Gong Y, Hua Y, Wang Z (2017) The role of propionic acid at diagnosis predicts mortality in patients with septic shock. J Crit Care 43:95-101

4. Steyerberg E (2008) Clinical prediction models: a practical approach to development, validation, and updating. Springer, Berlin

5. Smith GC, Seaman SR, Wood AM, Royston P, White IR (2014) Correcting for optimistic prediction in small data sets. Am J Epidemiol 180:318-324 